\title{
The Angular Momentum Structure of the Nucleon
}

\author{
Wolf-Dieter Nowak
}

DESY, D-15738 Zeuthen, Germany

\begin{abstract}
The proton spin budget is discussed. Results are presented from inclusive and semiinclusive deep inelastic scattering and from deeply virtual Compton scattering. They permit interpretations towards the determination of various contributions to the proton spin.
\end{abstract}

Keywords: Nucleon spin structure, QCD, spin-dependent DIS, spin-dependent nucleon structure function, generalized parton distributions

PACS: $12.38 . \mathrm{Qk}, 13.60 . \mathrm{Fz}, 13.60 . \mathrm{Hb}, 13.88 .+\mathrm{e}, 13.90 .+\mathrm{i}, 14.20 . \mathrm{Dh}$

\section{INTRODUCTION}

One of the most fundamental questions when studying the structure of the nucleon is how its spin $\frac{1}{2}$ is decomposed into the spins and orbital angular momenta of quarks and gluons. A milestone in the history of this field was the appearance in the late 1980s of an unexpected result of the European Muon Collaboration (EMC) [1, 2], which aroused great interest. Experimental advances in polarized-target technology in conjunction with the high degree of polarization of the $\mathscr{O}(100) \mathrm{GeV}$ polarized muon beam at the CERN SPS, had made it possible to measure the double-spin asymmetry $A_{L L}$ in the cross section of inclusive Deeply Inelastic Scattering (DIS) of leptons on nuclei. From this asymmetry the quark contribution $\Delta \Sigma$ to the nucleon spin was determined. It was found to be much smaller than the predictions within the widely accepted picture of three valence quarks constituting essential components of the nucleon. This finding was hence called a 'spin-puzzle'. Several new experiments were proposed and a wealth of theoretical studies initiated to solve it. Among the latter was another milestone, namely the finding [3] by Ji that the total angular momentum $J_{f}$ of quark species $f$ and that of gluons $\left(J_{G}\right)$ can be experimentally accessed in principle, by evaluating second moments of Generalized Parton Distributions (GPDs) [4] which had already been discussed 10 years before by Müller et al. [5]. GPDs were more recently interpreted by Burkardt [6] to describe simultaneously the transverse localization $b_{\perp}$ of a parton in the nucleon at a given fraction $x$ the parton carries of the longitudinal momentum $P$ of the nucleon. This description is sometimes also refered to as 3-dimensional picture of the nucleon.

In relativistic quantum mechanics, the spin of the nucleon can be decomposed into the total angular momenta of quarks and gluons. The 'Ji representation' [4] of this nucleon spin sum rule can be written in a covariant way as:

$$
\frac{1}{2}=J_{Q}\left(\mu^{2}\right)+J_{G}\left(\mu^{2}\right)
$$


with $J_{Q} \equiv \sum_{f} J_{f}$. In the context of Quantum Chromodynamics (QCD), the gauge theory of strong interactions, the individual terms in any decomposition of the nucleon spin depend on the factorization scheme and on the renormalization scale $\mu^{2}$. Results in different schemes are uniquely related to one another; mostly they are given in the so-called $\overline{\mathrm{MS}}$ scheme. The hard scale $\mu^{2}$ of the interaction can be chosen freely and independently of experiment. In lowest order perturbation theory (pQCD), lepton-nucleon scattering is treated in the one-photon-exchange approximation and $\mu^{2}$ is then identified with the virtuality of the exchanged virtual photon, $Q^{2} \equiv-q^{2}$, with $q$ being its 4 -momentum. The value of $Q^{2}$ is commonly chosen to be at least a few $\mathrm{GeV}^{2}$, a choice that is essential to keep all sorts of corrections as small as possible (most of them are suppressed by inverse powers of $Q$ ) when interpreting the data in terms of pQCD. Once an observable is known at one scale $Q_{0}^{2}$, QCD evolution equations [7] can be used to calculate it at another value $Q_{1}^{2}$. We note that no practical way is known to determine the gluon total angular momentum $J_{G}$. Still, it can be calculated as the difference $\frac{1}{2}-\sum_{f} J_{f}$.

With $\Delta \stackrel{(-)}{q}_{f}$ being the contribution from (anti)quark species $f$, the quark total angular momentum $J_{Q}$ in (1) can be replaced by the sum of the intrinsic quark spin contribution $\frac{1}{2} \Delta \Sigma \equiv \frac{1}{2} \sum_{f}\left(\Delta q_{f}+\Delta \bar{q}_{f}\right)$ plus the quark orbital angular momentum contribution $L_{Q} \equiv$ $\sum_{f} L_{f}$. Taking the $z$ (infinite momentum) axis as the quantization axis, this leads to:

$$
\frac{1}{2}=S_{z}=\frac{1}{2} \Delta \Sigma+L_{Q}+J_{G} .
$$

Here, only $\Delta \Sigma$ has a probabilistic interpretation as a parton number density.

Recent experimental results on $\Delta \Sigma$ from inclusive DIS will be presented below, followed by a description of the first steps towards a measurement of $J_{u}$ and $J_{d}$ in Deeply Virtual Compton Scattering (DVCS). Presently, no practical way is known to measure quark orbital angular momenta $L_{f}$ separately or their sum $L_{Q} \equiv \sum_{f} L_{f}$ as a whole. Hence the quark spin budget equation

$$
J_{Q}=\frac{1}{2} \Delta \Sigma+L_{Q}
$$

cannot be tested yet. Still, once $J_{u}$ and $J_{d}$ will be known, the valence quark orbital angular momentum $L_{u+d}$ can be obtained as the difference using (3).

Later in this article first results will be shown on $\Delta G$, the intrinsic gluon contribution to the nucleon spin. Then, also the difference $L_{G}=J_{G}-\Delta G$ could be calculated in principle [3]. However, while all observables discussed above are expectation values of gauge-invariant operators, for this difference no operator representation with any intrinsic relation to orbital angular momentum was identified yet, so that $L_{G}$ may remain a sterile definition (see [8, 9] for more detailed discussions). We note that both $L_{Q}$ and $L_{G}$ can be determined in QCD calculations on the Lattice (see, e.g., [10]).

Already back in 1989, as one of the first theoretical attempts to solve the spin puzzle, the alternative 'Jaffe-Manohar representation' [11] of the nucleon spin sum rule had been proposed, which is defined in light-cone $\left(A^{+}=0\right)$ or DIS gauge. It represents a decomposition of the $z$ projection of the nucleon spin:

$$
\frac{1}{2}=S_{z}=\frac{1}{2} \Delta \Sigma+\Delta G+\mathscr{L}
$$


where $\mathscr{L}$ is the parton (quark plus gluon) contribution to the nucleon spin, which can even be further decomposed into quark and gluon components: $\mathscr{L}=\sum_{f} \mathscr{L}_{f}+\mathscr{L}_{G}$. In decomposition (4) the (measurable) intrinsic gluon contribution $\Delta G$ to the nucleon spin appears explicitly and all terms have a probabilistic interpretation as a parton number density. No way to measure any of the orbital angular momenta $\mathscr{L}, \mathscr{L}_{f}$, or $\mathscr{L}_{G}$ is presently known nor can they be calculated in Lattice QCD. Still, $\mathscr{L}$ can be determined as the difference using (4).

Comparing the Ji representation (2) and the Jaffe-Manohar representation (4) of the nucleon spin sum rule (1) it can be seen that they have only $\Delta \Sigma$ in common, while all other components appear only in one or the other representation. The incommensurability of the two representations is discussed in [8, 9] by comparing the representations of the operators, the expectation values of which appear in one of the above discussed decompositions of the nucleon spin. It is concluded that, while both representations have their respective merits, according to present understanding and knowledge the components of the two representations must not be mixed because of incommensurate underlying operator representations.

\section{RESULTS FROM DEEPLY INELASTIC SCATTERING}

Doubly-polarized deeply inelastic scattering is sketched in the left panel of Fig. 1 , For inclusive DIS, in which only the scattered lepton in analyzed, the double-helicity asymmetry $A_{L L}$ is given by the DIS cross-section helicity difference, $\sigma_{L L} \equiv \frac{1}{2}\left(\sigma^{\vec{\digamma}}-\right.$ $\left.\sigma^{\rightrightarrows}\right)$, normalized by $\sigma_{U U} \equiv \frac{1}{2}\left(\sigma^{\rightleftarrows}+\sigma^{\rightrightarrows}\right)$, the unpolarized DIS cross section:

$$
A_{L L}=\frac{\sigma_{L L}}{\sigma_{U U}} \propto \frac{g_{1}}{F_{1}}
$$

Here ' $U$ ' and 'L' stand for unpolarized and longitudinally polarized (beam and target), and the single and double arrows denote the relative orientation of the spins of initial lepton and nucleon, respectively. As shown in (5), this asymmetry can be approximated by the ratio of $g_{1}$ and $F_{1}$, the spin-dependent and spin-averaged structure functions of the nucleon, respectively. They can both be expressed in terms of respective quark distribution functions.

The most recent result on $g_{1}$ was obtained by the HERMES collaboration [12]. In the right panel of Fig. 1 the $x$ dependence of $x \cdot g_{1}^{d}$ is shown together with earlier results. In DIS, $x$ is identified with the Bjorken variable $x_{B} \equiv Q^{2} /(2 P \cdot q)$. Using a few plausible assumptions, e.g. no significant contribution from the (unmeasured) small- $x_{B}$ region, the HERMES data set was used to accomplish the presently most precise experimental determination of the quark contribution to the nucleon spin [12]:

$$
\Delta \Sigma=0.330 \pm 0.011_{\text {theor. }} \pm 0.025_{\text {exp. }} \pm 0.028_{\text {evol. }},
$$

where 'exp.' includes statistical, experimental systematic and parameterization uncertainties, while the 'evol.' uncertainty arises from the necessity to evolve data to a common value of $Q^{2}$, here $5 \mathrm{GeV}^{2}$. 

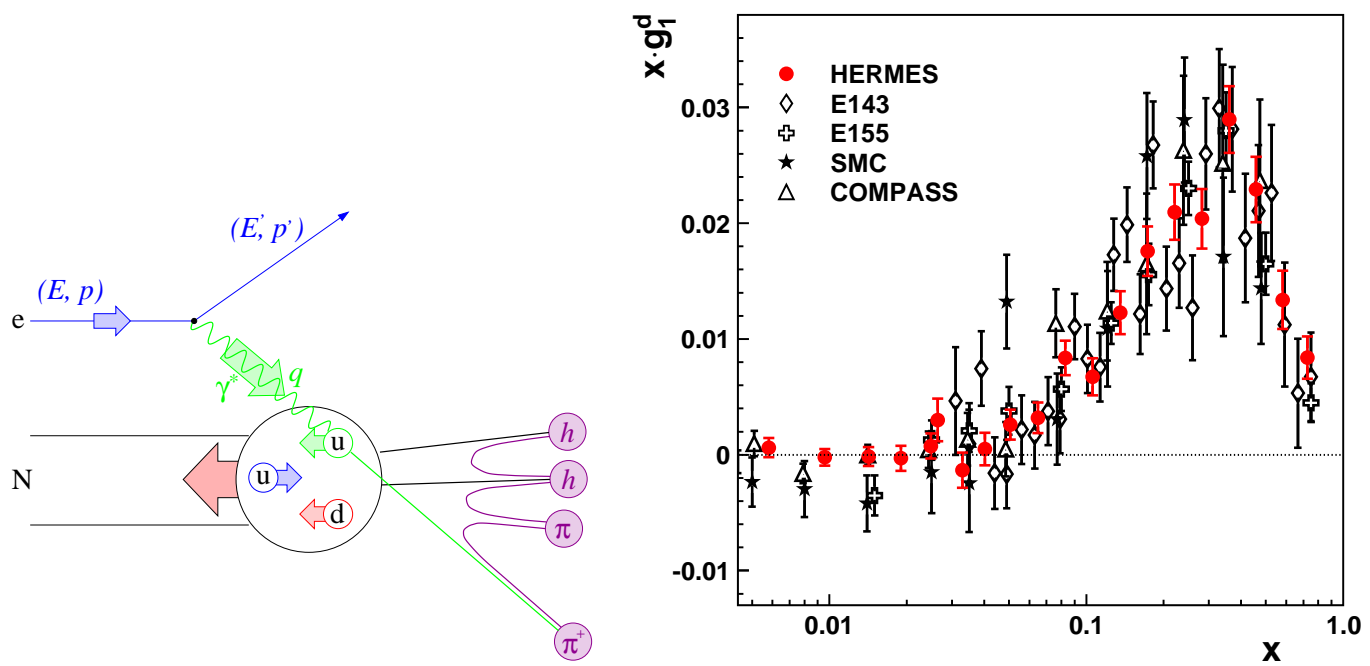

FIGURE 1. Left: Simplified schematic diagram of semi-inclusive DIS. Right: $x$ dependence of $x \cdot g_{1}^{d}$, as measured by HERMES [12], E143 [13], E155 [14], SMC [15] and COMPASS [16]. The figure is taken from [12].

There exists no result of comparable accuracy yet on $\Delta G$, the intrinsic gluon contribution to the nucleon spin, mainly because in inclusive DIS the virtual photon probes only quarks in the nucleon. The sensitivity to gluons in the nucleon can be enhanced when measuring hadrons in the final state, as those may be produced through additional QCD subprocesses, in particular photon-gluon fusion. Pioneering results were obtained in 'direct' measurements of the gluon polarization $\frac{\Delta G}{G}(x)$ in hadron photoproduction at very low $Q^{2}$, although only in the limited $x$ range covered by the data. Here, not only photongluon but also quark-gluon collisions occur because the hadronic content of the virtual photon gives rise to 'resolved-photon' subprocesses. Therefore, the resulting complicated mixture of subprocesses requires models in Monte Carlo simulations to describe the fractional contributions of the subprocesses within the experimental acceptance, so that such analyses were only performed in leading order and, moreover, the final result always remains model dependent. At these low values of $Q^{2}$ the hard scale of the subprocess is obtained by either selecting large transverse momenta of produced hadrons or by measuring charmed hadron production where the mass of the charm quark gives the hard scale.

The two presently most precise leading-order determinations of $\frac{\Delta G}{G}(x)$ were accomplished by COMPASS [16] analysing high- $p_{t}$ hadron pairs produced for $Q^{2}<1 \mathrm{GeV}^{2}$ :

$$
\left.\frac{\Delta G}{G}\right|_{\langle x\rangle \simeq 0.085}=0.016 \pm 0.058_{\text {stat }} \pm 0.055_{\text {syst }},
$$

and by HERMES [17] analysing high- $p_{t}$ single hadrons from quasi-real photoproduction:

$$
\left.\frac{\Delta G}{G}\right|_{\langle x\rangle \simeq 0.22}=0.071 \pm 0.034_{\text {stat }} \pm 0.010_{\text {sys-exp }} \pm_{0.105}^{0.127} \text { sys-Models } .
$$

In an alternative approach, sensitivity to the gluon content of the nucleon is found by fitting the $Q^{2}$ evolution of the moments of $g_{1}$ to the data, as this evolution involves 
gluon radiation by quarks and quark pair production by gluons. For such QCD fits none of the above described complications in direct measurements apply. Nevertheless, results on $\Delta G$ from earlier QCD analyses of only inclusive data showed quite large uncertainties, although predefined functional shapes were fitted and boundary conditions were imposed. The sensitivity to the gluon polarization in the nucleon and hence the accuracy of the fit can be improved by including data from semi-inclusive DIS (SIDIS) and doubly-polarized $p p$ collisions. In a very recent next-to-leading order QCD analysis [18], (semi-)inclusive data on $A_{L L}^{p, d}$ from COMPASs, JLAB and HERMES were combined with PHENIX and STAR $p p$ data on $A_{L L}^{\pi^{0}}$. Several results on $\Delta G$ are given at $Q^{2}=$ $10 \mathrm{GeV}^{2}$ for various ranges in $x$ and also for various ways to estimate the uncertainties. For the purpose of this article it appears sufficient to interpret their result as follows:

$$
\Delta G \approx-0.1 \pm 0.1
$$

The results (7) and (8) from the leading-order direct determinations of the gluon polarization are consistent with the fit result (9) on $\Delta G$. More precise results on the gluon contribution to the nucleon spin can be expected when more data from doubly-polarized $p p$ collisions at RHIC will become available.

Combining the DIS results (6) and (9), the Jaffe-Manohar representation (4) of the nucleon spin sum rule allows the determination of the parton orbital angular momentum in this representation:

$$
\mathscr{L} \approx 0.4 \pm 0.1
$$

\section{RESULTS FROM DEEPLY VIRTUAL COMPTON SCATTERING}

Exclusive production of a real photon from a virtual one, as illustrated in Fig. 2(a), is called Deeply Virtual Compton Scattering (DVCS). The competing Bethe-Heitler (BH) process, illustrated in Fig. 2(b), has an identical final state so that the two processes interfere on the level of amplitudes:

$$
\frac{d \sigma(\mathrm{eN} \rightarrow \mathrm{eN} \gamma)}{d x_{B} d Q^{2} d|t| d \phi} \propto\left|\mathscr{T}_{B H}\right|^{2}+\left|\mathscr{T}_{D V C S}\right|^{2}+\underbrace{\mathscr{T}_{B H} \mathscr{T}_{D V C S}^{*}+\mathscr{T}_{B H}^{*} \mathscr{T}_{D V C S}}_{I}
$$

Here $\phi$ is the azimuthal angle between the scattering plane, spanned by the incoming and outgoing leptons, and the production plane spanned by the virtual photon and the produced real photon (see right panel of Fig. 2). The Mandelstam momentum transfer between initial and final nucleon is denoted by $t$. The $\mathrm{BH}$ amplitude $\mathscr{T}_{B H}$ is exactly calculable in leading-order QED using the knowledge of the elastic nucleon Dirac and Pauli form factors $F_{1}$ and $F_{2}$. The DVCS contribution $\left|\mathscr{T}_{D V C S}\right|^{2}$ can be obtained by integrating over the azimuthal dependence of the cross section. It is sizeable at collider energies and on the few percent level at fixed-target energies, while the opposite holds for the contribution of the interference term I.

This interference term is of interest, as the measurement of its azimuthal dependence opens access to the complex-valued DVCS process amplitude. In this way both $\mathfrak{R}(\mathrm{I})$ and 


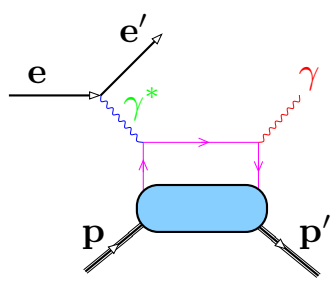

(a)

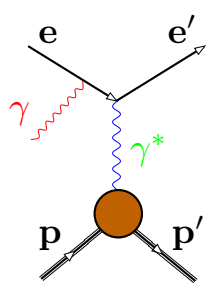

(b)

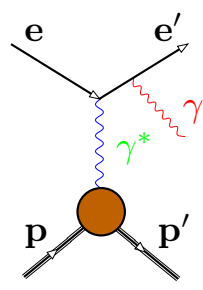

)

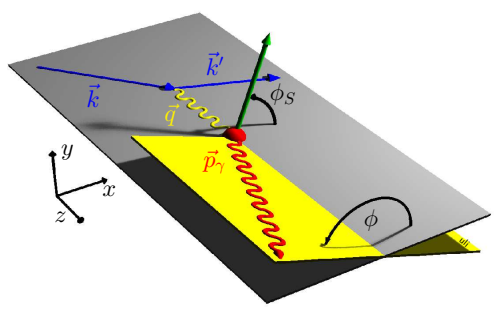

FIGURE 2. Left (a): 'Handbag' diagram in DVCS. Middle (b): Bethe-Heitler process. Right: Definition of azimuthal angles of produced real photon $(\phi)$ and target spin vector $\left(\phi_{S}\right)$.

$\mathfrak{I}(\mathrm{I})$ become accessible [19]. Using a $1 / Q$ expansion of the electroproduction cross section (11) the interference term can be represented in lowest order as a sum of azimuthal harmonics [20]. Here, besides important kinematic factors, the coefficients of the cos (sin) terms are real (imaginary) parts of certain Compton helicity amplitudes describing the DVCS process. In the interpretation of experimental data, only the nucleon-helicityconserving twist- 2 amplitude is considered, which can be written as a linear combination of $F_{1}$ and $F_{2}$ with Compton Form Factors (CFFs) $\mathscr{F}(\mathscr{F}=\mathscr{H}, \mathscr{E}, \widetilde{\mathscr{H}}, \widetilde{\mathscr{E}})$. These CFFs are flavour sums of convolutions of the respective GPDs $H^{f}, E^{f}, \widetilde{H}^{f}, \widetilde{E}^{f}$ with hard-scattering kernels. In such a convolution the GPD dependence on the momentum fraction $x$ is integrated out, so that - in contrast to DIS - DVCS does not permit a direct determination of the $x$ dependence of GPDs. Besides on $Q^{2}$ and $x$, GPDs also depend on $\xi$ and $t$ with the longitudinal momentum fraction $\xi$ describing the 'skewness' of the handbag diagram shown in Fig. 2(a). In the forward limit $\xi=0$, the quark taken out from the nucleon and the one put back into it carry identical momentum fractions and the GPDs $H^{f}$ and $\widetilde{H}^{f}$ become the spin-averaged and spin-dependent PDFs $q_{f}$ and $\Delta q_{f}$, respectively.

Various cross-section differences (or asymmetries) can be measured with respect to either beam spin or charge, or target polarization. They filter out certain parts of the interference term, i.e., they are represented by certain linear combinations of CFFs. Once the $t$-dependence of the GPDs $H^{f}$ and $E^{f}$ will have been determined, the second moment of their sum can be used to obtain the total angular momentum of quarks in the nucleon through the Ji sum rule [3]:

$$
J_{f}=\frac{1}{2} \lim _{t \rightarrow 0} \int \mathrm{d} x x\left[H^{f}(x, \xi, t)+E^{f}(x, \xi, t)\right] .
$$

Pioneering DVCS data from the HERMES experiment were analyzed [22] to determine cross section asymmetries with respect to beam spin and charge on one hand, and with respect to beam charge and target polarization on the other. In each case all relevant 'effective asymmetry amplitudes' were determined, which are the above mentioned coefficients of the $1 / Q$ expansion in azimuthal harmonics (for a detailed description see [22]). As an example, results from a 'combined' fit of the asymmetries with respect to beam charge and transverse target polarization are shown in Figs. 3 and 4, respectively. The data in Fig 4 are compared to calculations using various GPD models, see caption. In such models, the $u$ and $d$-quark total angular momenta $J_{u}$ and $J_{d}$ can be used as free parameters to parameterize the GPDs $E^{u}$ and $E^{d}$, respectively (see, e.g., [25]). 


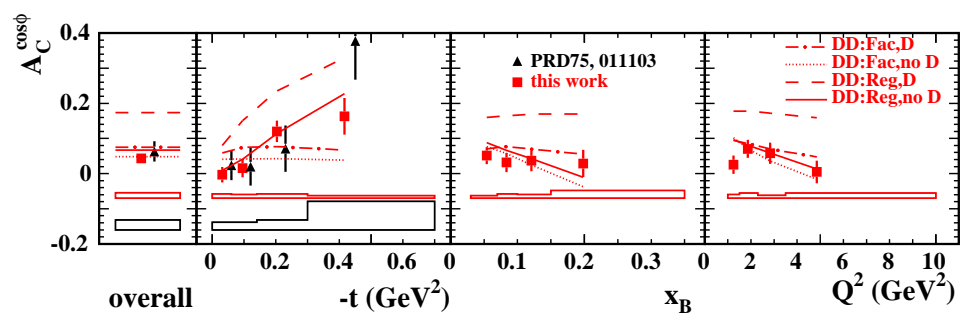

FIGURE 3. Azimuthal amplitude describing the dependence of the interference term on the beam charge $\left(A_{C}\right)$. The triangles (shifted right for visibility) represent previous results [21], while most recent data [22] are represented by squares. The error bars (bands) represent the statistical (systematic) uncertainties. The curves labelled 'DD' are calculations of variants of a double-distribution GPD model [23, 24] using $b_{v}=\infty$ and $b_{s}=1$ as profile parameters for valence and sea quarks. This figure shows the top panel of figure 4 of [22].

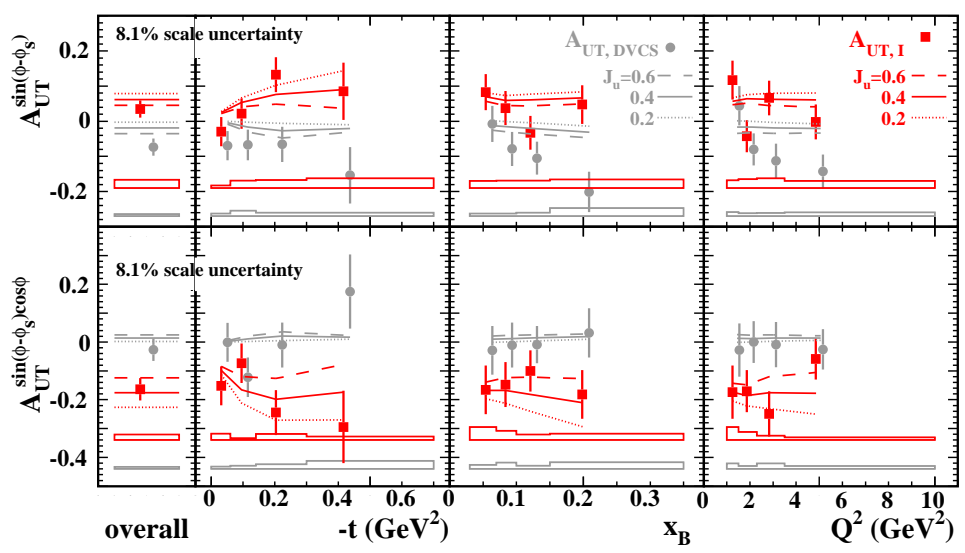

FIGURE 4. Asymmetry amplitudes describing the dependence of the squared DVCS amplitude (circles, $A_{U T, D V C S}$ ) and the interference term (squares, $A_{U T, I}$ ) on the transverse target polarisation. The circles (squares) are shifted right (left) for visibility. The error bars represent the statistical uncertainties, while the top (bottom) bands denote the systematic uncertainties for $A_{U T, I}\left(A_{U T, D V C S}\right)$, excluding an $8.1 \%$ scale uncertainty from the target polarisation measurement. The curves are calculations of the GPD model variant (Reg, no D) shown in the previous figure as a continuous curve, with three different values for the $u$-quark total angular momentum $J_{u}$ and fixed $d$-quark total angular momentum $J_{d}=0$ [25]. This figure shows the first two panels of figure 5 of [22].

The asymmetry amplitudes calculated from the GPD model of [23] were fitted to the HERMES data with transverse target polarization, resulting in the model-dependent constraint [22]:

$$
J_{u}+J_{d} / 2.8=0.49 \pm 0.17\left(\exp _{\text {tot }}\right) .
$$

This result shows that the data are already able to yield information on the total angular momenta of $u$ and $d$-quarks, while the limitations of the GPD model used prevent clear conclusions.

In leading-order approximation the values of the relevant (combination of) GPD(s) along the 'cross-over trajectory' $x=\xi$ contain the physical content of GPDs that is 
accessible in DVCS, while their shape in the $(x, \xi)$ plane is not accessible. In this context, predefined $(x, \xi)$ dependences used in available GPD models may be too inflexible and even ambigous [26]. At fixed $t$, CFFs satisfy dispersion relations [27, 28, 26] that allow the evaluation of the real part of a CFF from its imaginary part. By combining dispersion relation and operator expansion techniques convergence was proved for the conformal partial wave expansion of the VCS amplitude [29] and this new formalism was successfully applied to fitting DVCS observables in NNLO at low $x_{B}$. No global fits to all existing DVCS data exist yet.

The information on the $u$ and $d$-quark total angular momenta $J_{u}$ and $J_{d}$ that is presently available from DVCS experiments appears neither reliable enough to determine the gluon total angular momentum $J_{G}$ through (1) nor through (3) the valence quark orbital angular momentum $L_{u+d}$ in the Ji representation.

\section{ACKNOWLEDGMENTS}

I'm deeply indebted to M. Burkardt \& A. Miller for very exciting, fruitful and long discussions towards paper [8] without which the first section of this article would not have been possible. I'm grateful to A. Miller for reading this manuscript.

\section{REFERENCES}

1. J. Ashman, et al., Phys. Lett. B206, 364 (1988).

2. J. Ashman, et al., Nucl. Phys. B328, 1 (1989).

3. X.-D. Ji, Phys. Rev. Lett. 78, 610-613 (1997).

4. X.-D. Ji, Phys. Rev. D55, 7114-7125 (1997).

5. D. Mueller, D. Robaschik, B. Geyer, F. M. Dittes, and J. Horejsi, Fortschr. Phys. 42, 101 (1994).

6. M. Burkardt, Phys. Rev. D62, 071503 (2000), [erratum-ibid. D66 119903 (2002)].

7. G. Altarelli, and G. Parisi, Nucl. Phys. B126, 298 (1977).

8. M. Burkardt, A. Miller, and W. D. Nowak, to be subm. to Rep. Prog. Phys. ,arXiv:0812.2208[hep-ph].

9. M. Burkardt, and H. BC ,arXiv:0812.1605[hep-ph].

10. P. Hagler, et al., Phys. Rev. D77, 094502 (2008).

11. R. L. Jaffe, and A. Manohar, Nucl. Phys. B337, 509-546 (1990).

12. A. Airapetian, et al., Phys. Rev. D75, 012007 (2007).

13. K. Abe, et al., Phys. Rev. D58, 112003 (1998), [erratum-ibid. B429 414 (1998)].

14. P. L. Anthony, et al., Phys. Lett. B493, 19-28 (2000).

15. B. Adeva, et al., Phys. Rev. D60, 072004 (1999), [erratum-ibid. D62 079902 (2000)].

16. E. S. Ageev, et al., Phys. Lett. B612, 154-164 (2005).

17. P. Liebing, AIP Conf. Proc. 915, 331 (2007).

18. D. de Florian, R. Sassot, and M. Stratmann, Phys. Rev. D76, 074033 (2007).

19. M. Diehl, T. Gousset, B. Pire, and J. P. Ralston, Phys. Lett. B411, 193-202 (1997).

20. A. V. Belitsky, D. Mueller, and A. Kirchner, Nucl. Phys. B629, 323-392 (2002).

21. A. Airapetian, et al., Phys. Rev. D75, 011103 (2007).

22. A. Airapetian, et al., JHEP 06, 066 (2008).

23. M. Vanderhaeghen, P. A. M. Guichon, and M. Guidal, Phys. Rev. D60, 094017 (1999).

24. K. Goeke, M. V. Polyakov, and M. Vanderhaeghen, Prog. Part. Nucl. Phys. 47, 401-515 (2001).

25. F. Ellinghaus, W. D. Nowak, A. V. Vinnikov, and Z. Ye, Eur. Phys. J. C46, 729-739 (2006).

26. K. Kumericki, D. Muller, and K. Passek-Kumericki (arXiv:0805.0152 [hep-ph]).

27. L. Frankfurt, A. Freund, V. Guzey, and M. Strikman, Phys. Lett. B418, 345-354 (1998).

28. O. V. Teryaev ,arXiv:hep-ph/0510031.

29. K. Kumericki, D. Mueller, and K. Passek-Kumericki, Nucl. Phys. B794, 244-323 (2008). 